\title{
Application of Interactive Multimedia and Virtual Simulation Technology in Material Science \& Engineering Experiment
}

\section{Lu Han}

School of Materials Science and Engineering of Southwest Petroleum University, Chengdu, Sichuan, 610500, China

\begin{abstract}
In order to overcome various problems in traditional experimental teaching, a multimedia interactive teaching method was introduced in the School of Materials Science and Engineering of Southwest Petroleum University, combining modern teaching methods such as virtual simulation technology, students will be taught more intuitive and vivid experimental skills. Practice shows that all of those can both improve the active thinking and practical ability of students, and promote the development of experimental teaching in the material specialty, at the same time effectively improves the school' social reputation.

Keywords: Interactive multimedia teaching method; virtual simulation experiment; material science and engineering; experimental teaching

Citation: Lu Han, 2018. Application of Interactive Multimedia and Virtual Simulation Technology in Material Science \& Engineering Experiment. Advances in Material Science, 2(1): 5-8. http://doi.org/10.26789/AMS.2018.01.002

Copyright: Application of Interactive Multimedia and Virtual Simulation Technology in Material Science \& Engineering Experiment. (C) $2018 \mathrm{Lu}$ Han. This is an Open Access article published by Urban Development Scientific Publishing Company. It is distributed under the terms of the Creative Commons Attribution-Noncommercial 4.0 International License, permitting all noncommercial use, distribution, and reproduction in any medium, provided the original work is properly cited and acknowledged.
\end{abstract}

\section{Introduction}

With the rapid development of information technology, the mode of higher education in China has also undergone corresponding changes. A large number of information elements have been applied to experimental teaching, advanced multimedia technology, refreshing virtual simulation, and optical fiber network communication technology. The modern education model has become the new direction of China's education development ${ }^{[1-3]}$. Material science is a highly experimental subject, and experimental teaching is an important practical link in cultivating high-quality talents in materials ${ }^{[4,5]}$. However, there are some problems in traditional experimental teaching methods that limit the improvement of teaching effects and are not conducive to the cultivation of innovative spirit and practical ability talents ${ }^{[6]}$. In order to promote the "Excellent Engineer Education and Training Program" and further improve the quality of teaching, making full use of multimedia, computer network and virtual simulation technology to reform the teaching methods and content of materials science experiments has become an important part of the current laboratory construction.

\section{Problems in traditional experimental teaching}

\subsection{The experimental teaching method is single and the method is simple}

The teaching method used in traditional experimental teaching is a one-way transfer mode with teacher-centered and knowledge-based transmission. First, the teacher will explain, and then the students will follow the ready-made experiment steps to carry out the experiment step by step; or the teacher will explain the experiment principle first, and then the students will observe the teacher's demonstration experiment ${ }^{[7]}$. To a large extent, this experimental teaching model allows students to "passively" study, unable to think independently, and without their own ideas and creativity, which hinders the cultivation of students' innovative spirit and ability

\subsection{Some experiments have high cost or high safety risks}

The experimental equipment or reagents required for some experiments are relatively expensive. Considering the experimental cost, it cannot meet the operation needs 
of each student; the material processing equipment is mostly large-scale reinstallation equipment, which covers a large area and has high equipment value. It requires a lot of supporting equipment and runs. The cost is high. At the same time, the processing process involves high temperature, high pressure, and even arc electricity. Materials processing teaching experiments are carried out in the laboratory with physical objects alone. There are disadvantages such as high energy consumption, high pollution, high risks, and high safety hazards. Most of the students majoring in materials are studying China is mainly based on visits and internships, and the learning effect is not satisfactory.

\subsection{Analysis and testing experiments based on large-scale instruments and equipment}

The teaching effect cannot reach expectations. Largescale instruments are expensive. Most of them have only one or two sets, and the number of experimenters is large, which leads to a very low occupation rate per student. Therefore, students have extremely limited opportunities to use the computer during the test learning process. In addition, large-scale analytical instrument experiments involve The theories are mostly abstract and difficult to understand, and the experimental techniques are complex and varied. Teachers only teach the experimental process through words and a few pictures. They cannot show students the operation methods and technical points of various experimental techniques intuitively and vividly. At the same time, teachers demonstrate actions. At this time, due to the different viewing angles and different concentration levels of students, it is difficult for students to master all the standard operations and the essentials in one operation demonstration, which eventually leads to students not being able to master the knowledge points they have learned, and their interest in learning is not strong.

\subsection{Experiments under microscopic or extreme conditions cannot be carried out}

Like the effect of valence electron structure changes on the material structure and performance, the adsorption and desorption of the material surface interface and the characterization of chemical reactions, how to realize the design of materials from the atomic and molecular level according to the application and performance requirements of the materials, and the preparation of certain materials It is difficult to capture the changes in material characteristics and behavior under extreme conditions in the real experiments of the processing and processing processes. Such experiments cannot be carried out under the existing conditions in the laboratory, and related theoretical courses such as quantum mechanics, solid-state physics, and semiconductors. Physics and phase diagrams are often relatively abstract, and it is difficult for students to establish the relationship between formulas and applications, and it is difficult to learn and understand these key knowledge points and difficulties using traditional teaching methods.

\subsection{Students do experiments are limited by time and space}

Traditional experimental teaching is restricted by class hours and location. Although the laboratory opening policy is vigorously promoted at this stage, some largescale instruments and equipment are operated by trained personnel. In addition to the students' class time, various characterization analysis tests are being performed, so students can only be exposed to the course. For experimental equipment, once the course is over, students will not have the opportunity to enter the laboratory or access experimental equipment. For such courses as experimental teaching, once they leave the learning environment of the laboratory, students will feel very difficult and abstract in the process of autonomous learning ${ }^{[8]}$.

\section{Application of multimedia virtual simulation technology in material science experiments}

\subsection{Observation and analysis of the metallographic structure of the multimedia microscopic interactive system}

There are many metallographic experiment projects in the basic materials science and metal materials and heat treatment courses. In the experimental teaching, facing the characteristics of large number of experimenters, large amount of sample types and organization morphology information to be mastered, the college introduced a material microstructure experiment teaching system. The system connects the metallographic microscope to the computer, and directly displays the field of view under the metallographic microscope to the computer screen display, and uses the local area network to connect the computers of the entire laboratory together. By dividing the computer into the teacher side and the student side to achieve different Function. The teacher side can use the software to monitor the student side, and can also show the typical metallographic atlas to the whole class for viewing, and give targeted explanations, so as to better help students master different forms of metallographic organization. The student terminal can not only collect metallographic images, but also measure the average grain size of metals. Through this system, not only real-time display, full-pro- 
cess monitoring, timely feedback and other functions can be realized ${ }^{[9]}$, but also the enthusiasm of students' experiments and teaching effects have been significantly improved. With the help of this system, students of the School of Materials Science and Engineering of Southwest Petroleum University can not only consolidate their in-class knowledge through open experiments of college students, but also participate in many national competitions such as the National Metallographic Skills Competition and the Material Comprehensive Skills Competition. Good results for winning the first prize in four years.

\subsection{Record operation video of large-scale analytical equipment}

The School of Materials Science and Engineering recorded the experimental operation techniques of largescale analytical instruments with a value of more than 300,000 and a high number of operating hours into a series of videos to replace the teacher's demonstration in class. In traditional experimental teaching, in the process of demonstrating operation to students, some students cannot keep up and cannot see clearly, especially the parameter settings of the computer screen interface and mouse operations cannot be recorded in detail. In general, the operation of the instrument is demonstrated Irreversible, generally only performed once, which is not conducive for students to fully grasp the operation process of the instrument. By playing the recorded video of the instrument operation, the time for students to test the experiment is flexibly increased. Students can preview before class. At the same time, the teacher can pause the video at any time during the explanation process, repeat key actions or change the operation process. The precautions in this article are emphasized to students, so that they can remember and understand the details of the operation process more clearly. Among them, one of the requirements for the professional master's training in the School of Materials Science and Engineering is that one must master the operation skills of a large-scale equipment and obtain an operation certificate.

Through the recorded large-scale instrument operation video, students can choose the type of study according to their professional direction and hobbies, and they can also study and explore as an extracurricular hobby. At the same time, the recorded operation video can also be used as a post-production virtual simulation platform for large instruments and equipment.

\subsection{Open virtual simulation experiment courses}

Virtual simulation technology can simulate real ex- periments or working conditions, complete various predetermined experimental projects or engineering projects, obtain real and intuitive experimental results, show invisible structures or principles, and have strong controllability, environmental protection, energy saving, and breakthroughs. The advantages of traditional teaching time, space and experimental conditions are the important direction of the development of many university laboratories in China and internationally in recent years ${ }^{[10,11]}$.

The School of Materials Science and Engineering carried out related research and exploration as early as 2004 . With the support of the school, it carried out a research on the experimental teaching project of casting molding simulation in 2005 , using virtual reality technology, simulation technology, multimedia technology, human-computer interaction technology, Database technology and network technology are combined with material science and engineering practice ${ }^{[12]}$ to carry out the fluid flow process in the high-pressure casting molding process, the high-pressure molding process of large forging parts, the solid flow of metal in the mold, and the temperature during the casting process. With simulation experiment teaching such as stress change simulation, students can use the molding simulation software to complete the influence of liquid molding process parameter changes on the liquid filling process, temperature field, and stress field, predict casting defects, and then change the casting process to correct the molding. Analyze the influence of each factor on the casting molding process, and combine the physical tests such as "Smelting and molding of aluminum alloy", "Sand performance evaluation and modeling", etc., to deepen the understanding of the liquid molding process and cultivate students' innovative thinking consciousness And engineering practice ability.

In 2012, the "Material Processing Simulation and Simulation" course was opened to explore the experimental teaching project of plastic forming simulation and simulation, which solved the teaching difficulties of the difficulty of observing and copying the plastic forming process and the evolution of the "four elements" of materials in the material forming process. In 2014, the welding numerical simulation course was added and related experimental projects were opened. Through the computer simulation of the welding process, the influence of various factors in the welding process on the welding quality can be quantitatively analyzed, and the welding heat transfer and the fluid power in the welding pool can be analyzed. Research, the heat and mass transfer process of the welding arc, the prediction of welding deformation and residual stress ${ }^{[13]}$.

At the same time, using the virtual simulation experiment platform, the teacher guided the relevant students' 
graduation thesis and extracurricular open experiments, and also instructed the students to actively participate in various virtual simulation competitions, which expanded the students' practical space and enhanced their understanding of professional theoretical knowledge and production practices.

\section{Conclusion}

The training of talents in materials discipline involves the design, preparation, processing and performance of materials, which is a typical combination of science and engineering. It not only requires students to have a solid foundation and broad knowledge, but also requires students to have strong practical skills. However, the traditional practical teaching methods are far from meeting the requirements for the cultivation of innovative talents. With the help of modern multimedia numerical technology, virtual simulation and computer network technology, intuitive and vivid images and colorful animations are used in various experimental techniques. The basic principles and experimental procedures can attract more students' attention and stimulate students' curiosity, while avoiding the disadvantages of high energy consumption, high pollution, high risks, and high potential safety hazards, allowing students to use the processing virtual simulation based on the knowledge learned in theory Technology, virtual reality combined with simulated material processing behavior, practice simulation of the change process of organization and performance in processing, can effectively improve the teaching effect. Therefore, fully tapping and exerting the advantages of modern information technology in materials science experiment teaching can improve students' understanding of abstract knowledge, mastery of experimental skills, and enhancement of practical ability, which is more conducive to cultivating students' innovative consciousness and innovative spirit.

\section{References}

[1] Guo Chenming, Fu Minggang, Guo Liying. Application of video teaching in surgical clinical teaching $[\mathrm{J}]$. Continuing Medical Education, 2014(05): 49-50.

[2] He Xiujuan. Practice comparison and thinking between multimedia teaching and traditional classroom teaching $[\mathrm{J}]$. Experimental Technology and Management, 2005(12): 94-96.

[3] Wang Jing, Yang Jinping, Wang Chunmei. The appli- cation of virtual simulation in the practical teaching of inorganic non-metallic materials[J]. Experimental Technology and Management, 2015(07):107-108.

[4] Li Liangliang, Zhao Yuzhen, Li Zhengcao, et al. Construction of Virtual Simulation Experimental Teaching Center for Materials Science and Engineering $[\mathrm{J}]$. Experimental Technology and Management, 2014(02): 5-8.

[5] Gao Yuan, Xi Shengqi, Sun Qiaoyan, et al. Application of virtual simulation technology in materials science experiment teaching [J]. China Modern Educational Equipment, 2016(03): 94-98.

[6] Chen Rongrong, Sun Yiding, Wei Dongsheng, et al. Multimedia virtual simulation teaching method should be used in microbiology experiment[J] Research and Exploration in Laboratory, 2015(11): 194196.

[7] Chen Yuanhong, Tang Huaying, Wei Hongyu, et al. Talk about the necessity of building a virtual laboratory of medical immunology[J]. Journal of Youjiang Medical College for Nationalities, 2011(05):699-701.

[8] Liu Zhengwei, Bian Yahong, Zhang Li. Design and development of virtual experiment training system for large-scale pharmaceutical analysis instruments[J]. Chinese Medical Education Technology, 2014(03):280-284.

[9] Liu Changhong, Ai Yunlong, Deng Keming. The reform of metallographic experiment teaching based on multimedia microscopy interactive system[J]. Experimental Technology and Management, 2008(05): 9094.

[10] Department of Higher Education, Ministry of Education. Notice on the construction of a national virtual simulation experiment teaching center [Z].2013.

[11] Yan Jihong, Zhang Fenyang. Development of simulation optimization module for virtual reconfigurable manufacturing system $[\mathrm{J}]$. Laboratory Research and Exploration, 2013(07): 81-86.

[12] Shen Wenzhu, Wang Bin, Yi Feng, et al. Construction and practice of virtual experiment platform for material forming $[\mathrm{J}]$. University Education, 2017(05): $72-73$.

[13] Lu Dongli, Zhang Tao. The application of virtual simulation technology in the comprehensive experiment of material control specialty[J]. Education Teaching Forum, 2015(11):226-227. 